Revue des patrimoines

\title{
Mémoire architecturale au miroir de la France et de l'Allemagne. L'ancienne ambassade de France en Sarre 1945 - 1955 / 2018
}

The former French Embassy in Saarbrïcken, Saarland, a bridge to Europe, 1945-1955

\section{Eva Mendgen}

\section{OpenEdition}

\section{Journals}

Édition électronique

URL : http://journals.openedition.org/insitu/20250

DOI : $10.4000 /$ insitu. 20250

ISSN : $1630-7305$

\section{Éditeur}

Ministère de la culture

\section{Référence électronique}

Eva Mendgen, « Mémoire architecturale au miroir de la France et de l'Allemagne. L'ancienne ambassade de France en Sarre 1945 - 1955 / 2018 », In Situ [En ligne], 38 | 2019, mis en ligne le 15 février 2019, consulté le 02 mai 2019. URL : http://journals.openedition.org/insitu/20250; DOI : 10.4000/insitu.20250

Ce document a été généré automatiquement le 2 mai 2019.

\section{c) (†)}

In Situ Revues des patrimoines est mis à disposition selon les termes de la licence Creative Commons Attribution - Pas d'Utilisation Commerciale - Pas de Modification 4.0 International. 


\section{Mémoire architecturale au miroir de la France et de l'Allemagne. L'ancienne ambassade de France en Sarre 1945 - 1955 / 2018}

The former French Embassy in Saarbrïcken, Saarland, a bridge to Europe, 1945-1955

\section{Eva Mendgen}

« Fasse le ciel qu'il se trouve quelque citoyen assez

\section{« Une région-charnière » au centre de l'Europe}

2 Cet article est consacré à l'ambassade de France en Sarre, un bâtiment au destin singulier qui fait partie du patrimoine non seulement architectural mais aussi politique et culturel de la Sarre, dont l'avenir est aujourd'hui incertain. Si l'on peut se réjouir de ce qu'elle attire depuis peu l'attention des chercheurs, cette ambassade reste toutefois un héritage dont la portée est largement méconnue (fig. 1). 
Figure 1

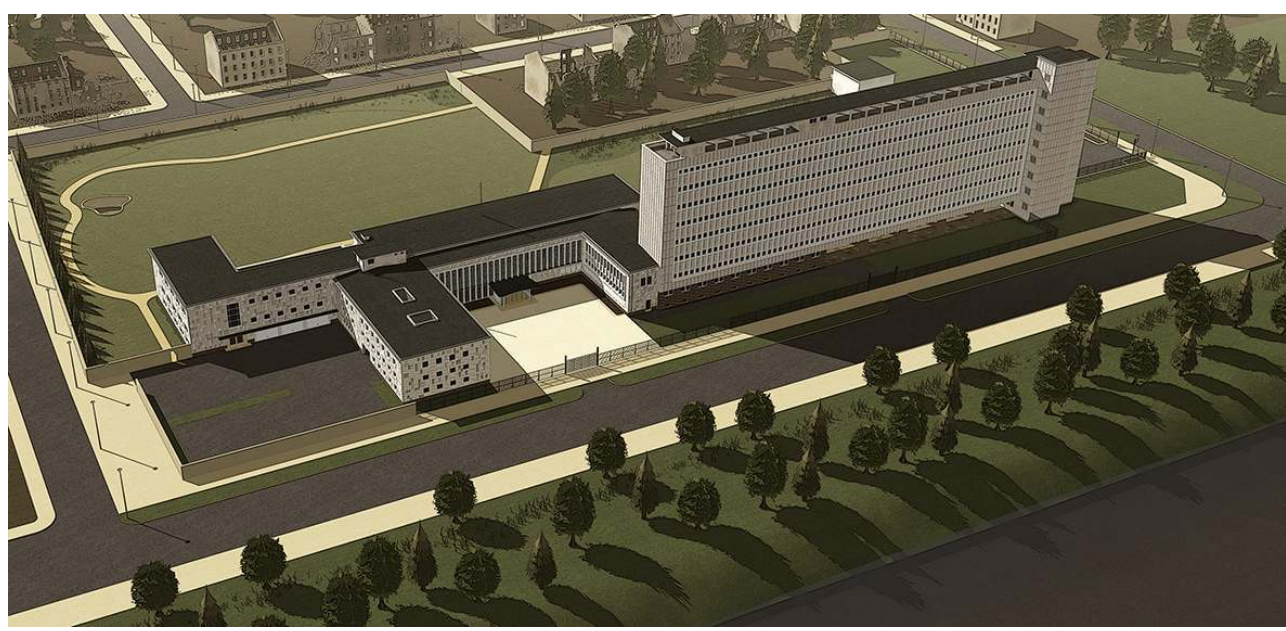

Interprétation 3D par Maxime Santiago, 2017. En collaboration avec Alexandra Schlicklin, Sylvie Grimm-Hamen et Norbert Mendgen dans le cadre du projet de recherche portant sur la construction d'un portail consacré aux «Cultures transfrontalières / Räume der Grenze », (université de Lorraine et CNRS en coopération avec régiofactum, réseau culturel de la Grande Région / Großregion SaarlandLorraine-Luxemb(o)urg-Rheinland-Pfalz-Wallonie(n).

(c) Maxime Santiago.

3 L'une des raisons de cette méconnaissance tient au fait que les études qui lui sont consacrées ne prennent pas suffisamment en considération l'environnement frontalier et biculturel de l'édifice. On peut également avancer que l'ambassade n'a pas encore fait l'objet d'une analyse profonde ni d'une recherche scientifique qui rende justice à la complexité de l'histoire de sa construction, de son aménagement intérieur y compris son mobilier, de sa définition et de sa situation dans l'espace de ville. Jusqu'à aujourd'hui, ces considérations n'ont été envisagées qu'à leur surface.

Contrairement à ce que l'on peut fréquemment lire sur le sujet, les acteurs politiques et culturels engagés dans ce projet novateur et ambitieux au sortir de la Seconde Guerre mondiale furent à la fois français et sarrois et, pour des raisons différentes, ceux-ci se virent comme des pionniers de l'Europe. Le contexte singulier de cette ambassade est en effet celui de la ville de Sarrebruck, devenue la capitale du Land de Sarre après l'unification avec la RFA en 1957/1959. La Sarre et Saarbrücken - que les Français orthographient volontiers « Sarrebrück » - sont une région et une ville industrielles à la frontière franco-allemande qui furent longtemps l'enjeu de visées nationalistes, avant de devenir après-guerre des lieux emblématiques du projet européen porté par Robert Schuman et par les personnalités politiques de l'après-guerre en Sarre, Gilbert Grandval et Johannes Hoffmann.

5 Vue à travers ce prisme européen, l'ancienne ambassade de France en Sarre gagne une dimension nouvelle: elle apparait comme le témoin singulier d'une expérimentation à grande échelle sur la vocation européenne de cette région. Dans son premier numéro de mai-juin 1945, la revue parisienne L'Architecture d'aujourd'hui attribuait déjà aux régions bilingues situées le long de la frontière franco-allemande une importance particulière. En devenant une zone française d'occupation, la Sarre devenait de fait, comme les autres territoires annexés et en grande partie détruits, une zone de reconstruction à la fois matérielle et immatérielle, et le terrain privilégié d'un nouveau départ permettant de 
surmonter les anciens combats idéologiques, et qui devait se réaliser dans le cadre d'un « planning moderne » qui restait encore largement à définir²

\section{« Esprit contemporain »}

«C'est que si le passé est séparé du présent par des obstacles, des collines, des montagnes, des cassures, des différences, il a, pour le rejoindre, ses routes, ses chemins, ses infiltrations : il flotte autour de nous, méconnu et insinuant, et, sans trop le savoir, nous y sommes englués. » (Fernand Braudel $)^{3}$

7 Ce que l'historien Fernand Braudel constatait à propos de l'histoire de France vaut aussi tout particulièrement pour celle de la Sarre. Pour celui qui la regarde de loin, elle donne d'abord le sentiment d'être un espace étrangement anhistorique. Mais quand on y vit, on constate que les conflits du passé récent ne sont jamais loin. Les traces laissées notamment par les référendums de 1935 et 1955 - dans des circonstances tout à fait différentes, la population a eu à se prononcer deux fois sur sa réunification avec l'Allemagne ${ }^{4}$ - sont toujours là, de façon latente.

La Sarre - ou mieux encore le Saarland - est une construction politique qui n'existe en tant que telle que depuis le $\mathrm{xx}^{\mathrm{e}}$ siècle ${ }^{5}$. Sa population a toujours été soupçonnée, par les Allemands comme par les Français, de vivre dans un entre-deux et de ne pas avoir d'appartenance nationale clairement définie. Selon les cas, les Sarrois passaient pour être soit imprévisibles soit rapidement assimilables. Pendant près de deux siècles, la France rivalisa avec l'Allemagne pour conquérir cette région que ses riches gisements de charbon prédisposaient à devenir un centre de l'industrie de l'armement, mais que sa situation frontalière rendait particulièrement vulnérable.

Entre le 15 décembre 1947 et le 23 octobre 1955, la Sarre eut pour la première fois l'opportunité de devenir un «foyer de la culture européenne » et un « lieu de rencontre de l'Europe ", et cela sous les auspices de la France. Dans la brochure de candidature de Sarrebruck comme capitale de la Communauté européenne du charbon et de l'acier $(\mathrm{CECA})^{6}$, on peut lire en 1953 que la ville "y voit un honneur, mais aussi un encouragement à poursuivre la nouvelle voie dans laquelle elle s'est engagée $»^{7}$. Trop d'études scientifiques négligent la dimension européenne de cet " esprit contemporain " revendiqué d'abord dans le plan de reconstruction régional, avant de présider à la fondation d'une université franco-allemande à Sarrebruck, appelée à devenir la première université européenne, selon les termes de la convention culturelle bilatérale conclue entre la France et l'État de la Sarre en 1948. Dès le début, l'accent mis sur le bilinguisme et les approches comparatives, dans un établissement qui était alors une antenne de l'université de Nancy, devait créer les conditions d'un rapprochement entre les peuples et former des pionniers de l'Europe (fig. 2). Dans le même esprit, on fonda aussi un centre des métiers d'art où l'on formait des artisans et des techniciens de la reconstruction, tels les architectes ${ }^{8}$. 


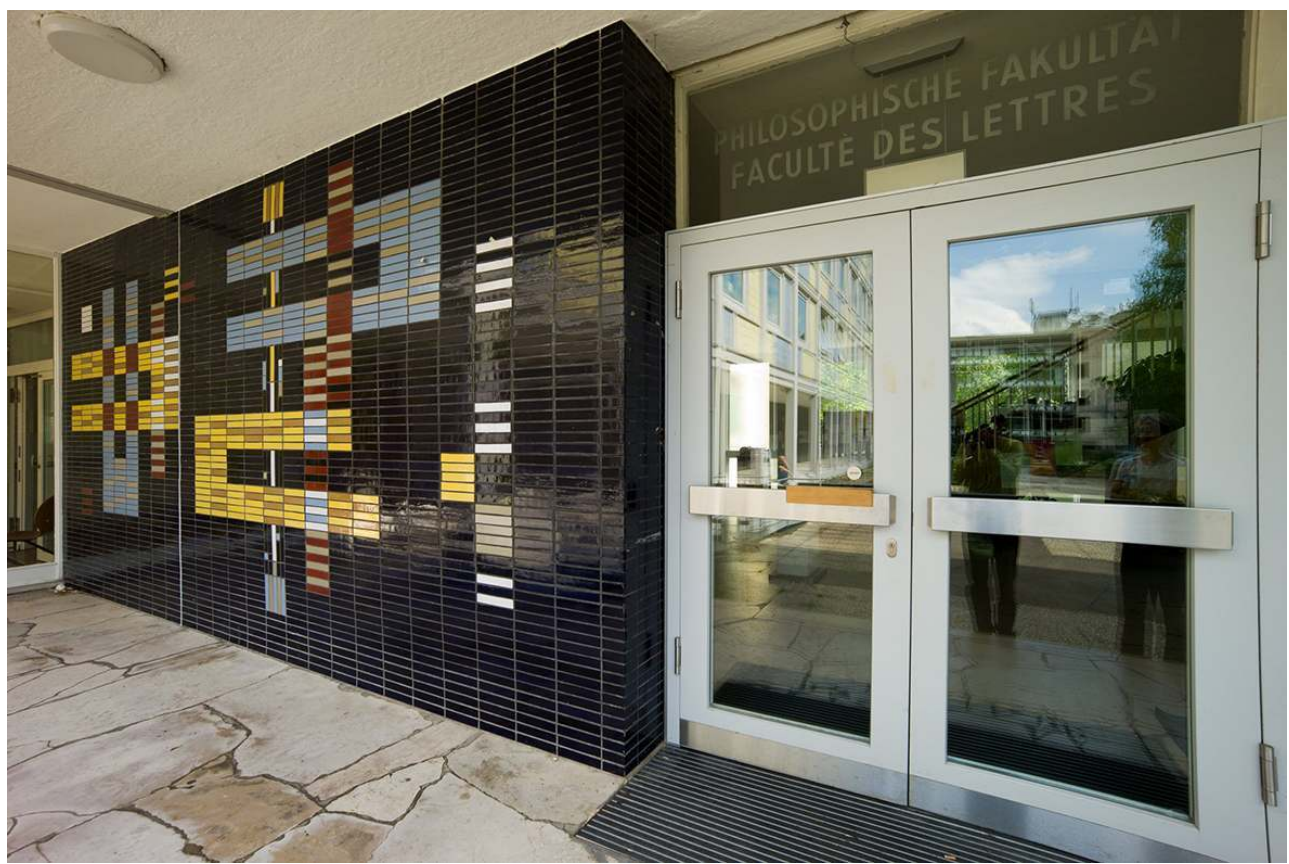

Faculté des Lettres, Université de la Sarre, Sarrebruck. Architecte : André Remondet, artiste : Wolfram Huschens (sarrois), 1954-1955 ( ?).

Phot. die arge lola, 2012. (c) die arge lola/regiofactum.

10 L'Europe allait offrir ainsi un nouvel horizon à une région longtemps livrée aux ambitions de la France ou de l'Allemagne'.

\section{«Architectes associés »}

11 L'ancienne ambassade de France à Sarrebruck est le dernier témoin emblématique de la vocation européenne de la Sarre entre 1945 et 1955, pendant ce que l'on continue généralement d'appeler "die französische Zeit » («l'époque française »). D’un point de vue juridique, l'édifice est en effet le fruit d'une collaboration des " architectes associés » franco-sarrois Georges-Henri Pingusson, Bernhard Schultheis et Hans Bert Baur ${ }^{10}$. Il a été conçu et érigé entre juillet 1950 et janvier 1955.

Son « commanditaire » français fut Gilbert Grandval (1904-1981), qui avait été colonel dans l'armée de la Résistance (CdlR) dans l'Est de la France avant de devenir gouverneur militaire, haut-commissaire puis ambassadeur de France en Sarre (1945-1955) (fig. 3). 


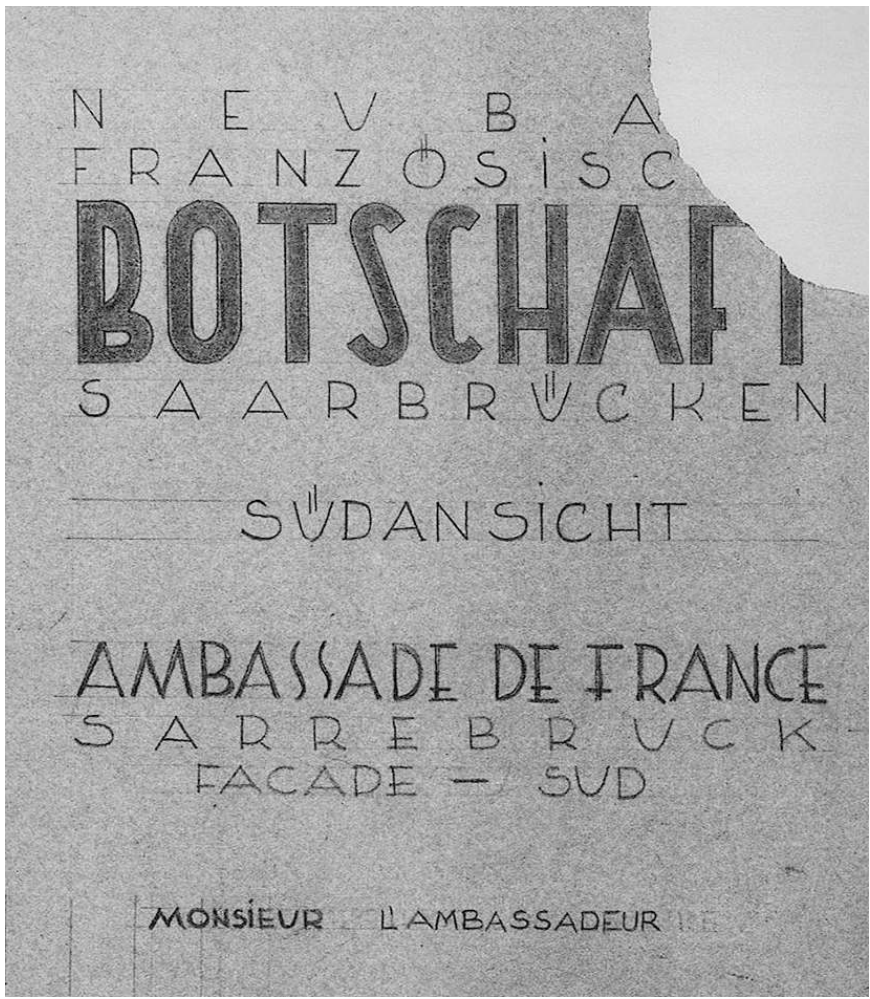

Détail du plan de construction, septembre 1951. Collection privée.

Baur, un architecte formé initialement dans la tradition du Neues Bauen ${ }^{11}$ à Stuttgart et qui exerça dans plusieurs bureaux d'architectes en Allemagne et en Autriche, a travaillé une première fois pour Grandval dans le cadre de la reconstruction du château du Halberg (fig. 4), situé sur les hauteurs de la vallée de la Sarre, en tant que responsable de l'aménagement de cette première résidence du gouverneur militaire ${ }^{12}$. C'est au plus tard à cette époque que Baur collabora pour la première fois avec Pingusson qui avait pour sa part dessiné les plans du bureau de Grandval dans sa première résidence, le « Halberg Schloss $\aleph^{13}$. Les transformations apportées par Baur ne sont pas sans évoquer la future ambassade qui sera édifiée à partir de 1950 dans le centre de la ville, sur la rive du fleuve. Schultheis, quant à lui, était depuis 1948 le premier président de la chambre d'architecture de la Sarre. 


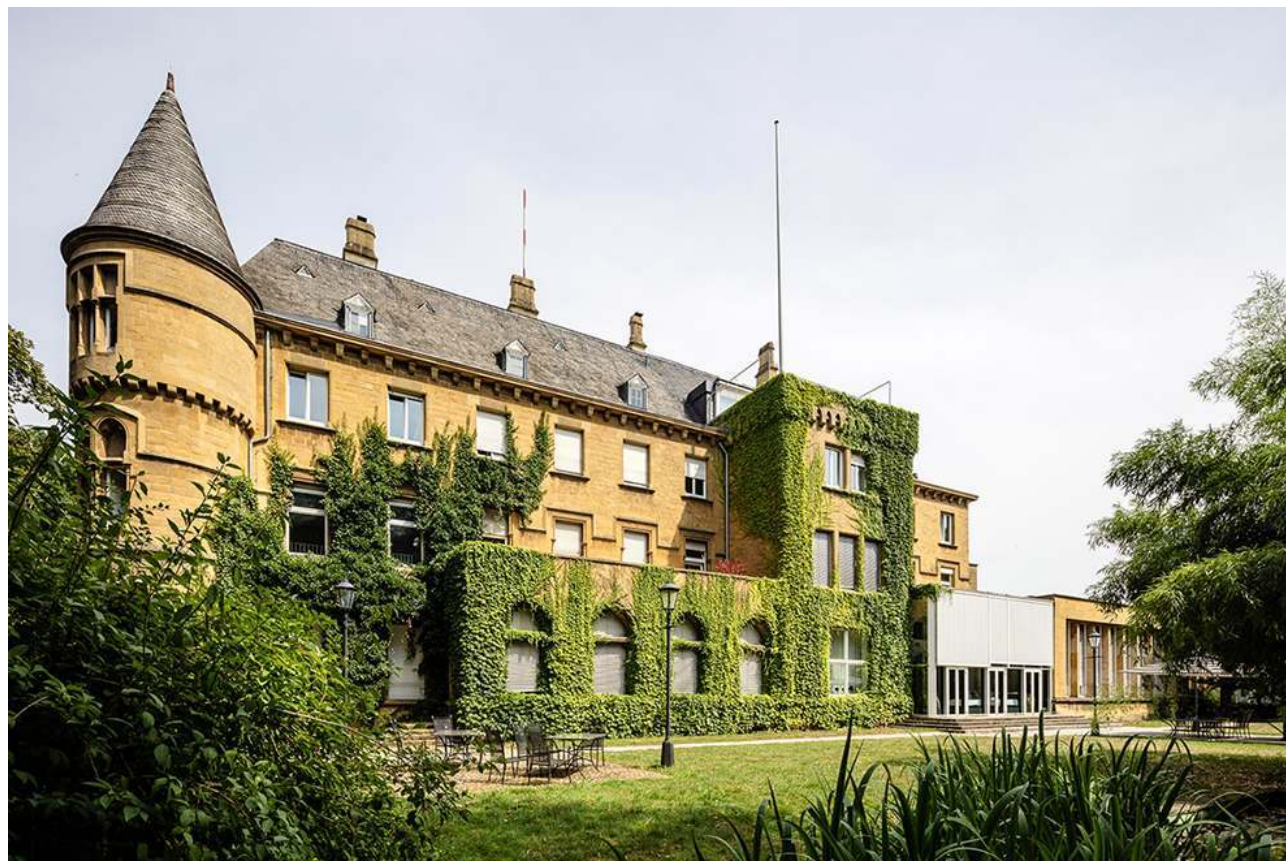

Château du Halberg, résidence de l'industriel sarrois Ferdinand von Stumm. Architectes : Edwin von Oppler, 1877 (dans l'esprit de Viollet-le-Duc) / 1945-1946 (?). Reconstruction comme résidence du gouverneur militaire Gilbert Grandval par l'architecte Hans Bert Baur.

Phot. die arge lola, 2016. (c) die arge lola/regiofactum.

\section{«Un pont sur la Sarre »}

14 Le propriétaire du terrain sur lequel fut construit l'ambassade était l'État de Sarre, représenté par Johannes Hoffmann, un ancien journaliste sarrois qui devint le 15 décembre 1947 le premier ministre-président de la Sarre après être rentré en août 1945 d'un exil au Brésil, où il avait cherché à échapper aux persécutions du $3^{\mathrm{e}}$ Reich.

Le bâtiment avait de ce fait un statut hybride : financé à la fois par la France et la Sarre, il appartenait à la Sarre alors indépendante, mais était dévolu autant à la représentation de la France qu'à celle de la Sarre en Europe. Le temps a malheureusement manqué pour installer cette vision dans les esprits, dans la mesure où, dès 1955, les enjeux devenaient autres.

Le jour même de la nomination de Johannes Hoffmann, la Constitution de la Sarre prit effet. Son préambule s'adressait au peuple sarrois, le "saarländisches Volk», et il stipulait que «sa mission est de constituer un pont pour l'entente entre les peuples / dessen Sendung es ist, eine Brücke zur Verständigung der Völker zu bilden $»^{14}$. L'image évoquée ici allait figurer dans le drapeau et les armoiries de ce nouvel État ${ }^{15}$ (fig. 5). 
Figure 5

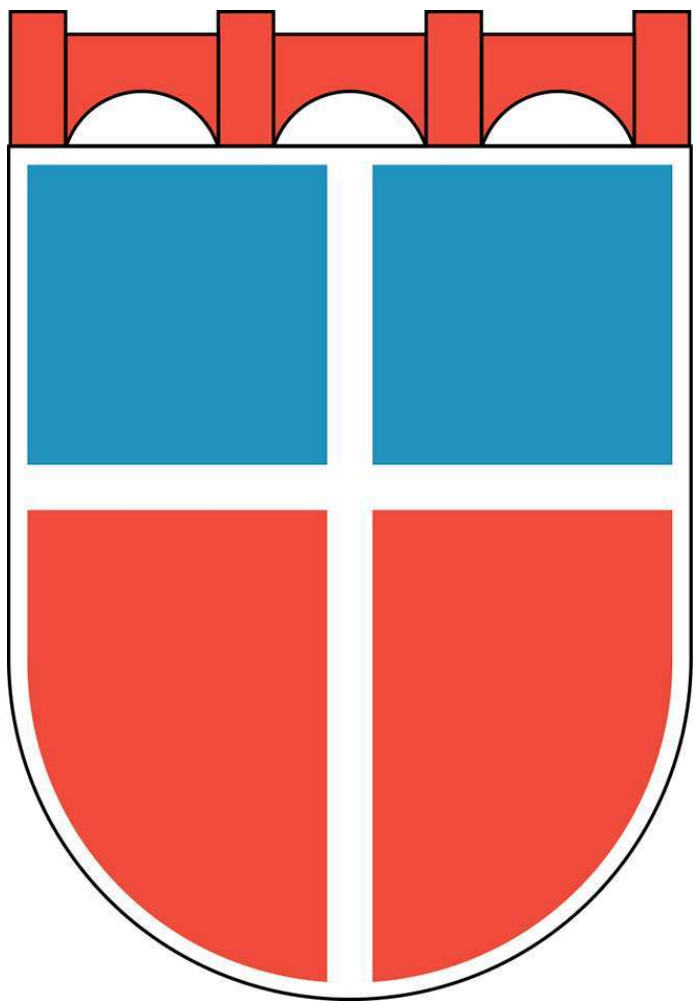

Armes de la Sarre, 14 décembre 1948. Loi relative aux Armes de la Sarre du 14 déc. 1948, Amtsblatt des Saarlandes Nr. 95 / 1948, page 1549. Voir: http://www.saar-nostalgie.de/.

Elle se retrouve aussi dans l'image de "région-charnière » qu'il s'agissait de faire de la Sarre, selon le « plan régional » publié en mai 1947 dans l'étude La Sarre. Urbanisme. 1946 ${ }^{16}$, un premier manifeste plaidant pour la réinterprétation et la réorganisation de ce territoire dans le respect de ses caractéristiques politiques (militaires), économiques et culturelles.

Grandval et Pingusson, pour qui ce plan restait toujours d'actualité au moment de la construction de l'ambassade, quelques années plus tard, s'accordaient tous deux à voir la Sarre comme «l'un des meilleurs territoires d'Europe ${ }^{17}$ ». Pour Pingusson, l'urbanisme moderne était capable de redéfinir le vivre ensemble et de l'adapter aux besoins de l'homme contemporain, de faire sortir des ruines « d'immenses sources de bonheur et de richesse $^{18}$ ». Son ambition fut de contribuer à donner une identité européenne moderne à ce territoire " entre deux cultures ", prédestiné à devenir « un élément de transition et d'harmonisation ${ }^{19}$ ".

Un coup d'œil sur le plan de Sarrebruck montre que Pingusson avait envisagé un nouveau centre-ville avec une "place principale» sur la rive droite de la Sarre ${ }^{20}$, qui devait s'ouvrir et se prolonger sur l'autre rive du fleuve (fig. 6). C'est exactement là que s'élèvera l'ambassade peu de temps après, sur la rive gauche du fleuve, du côté de la France. L'édifice érigé le long de la Sarre bordait la "route de l'Europe» entre Luxembourg et Strasbourg"21. Pour Pingusson, celui-ci devait incarner «la volonté de tisser des liens d'amitié féconds et durables (sic!) ${ }^{22}$ ». Le nom même de la ville, Sarrebruck (Saarbrücken), selon lui, contenait en germe sa vocation à être un " pont sur la Sarre ». Dans le même temps, le bâtiment était censé ouvrir aussi une perspective sur la 
ville elle-même et sur les nouvelles infrastructures politiques, comme le Parlement (« Landtag $»)^{23}$.

Figure 6

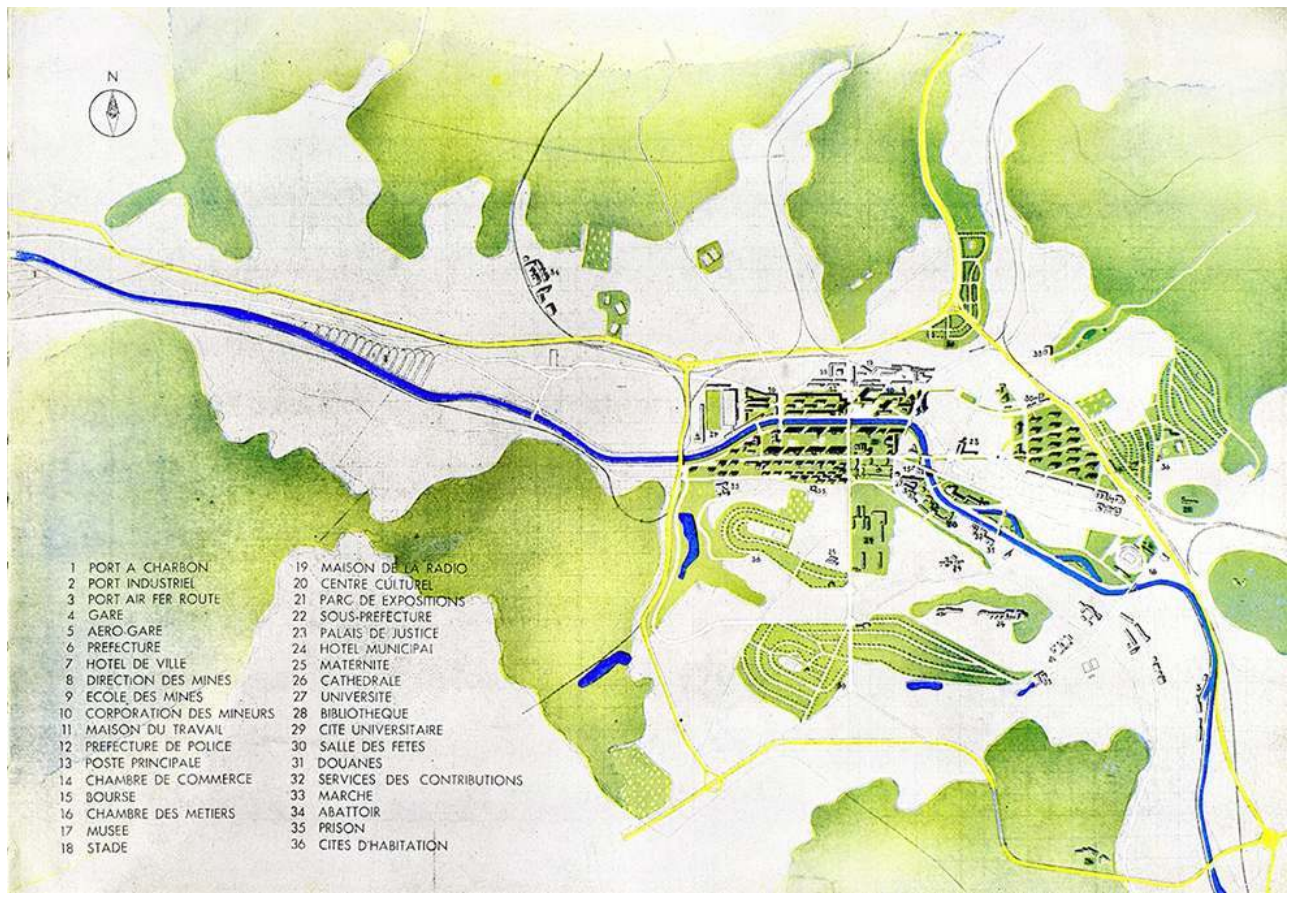

Gouvernement militaire de la Sarre, section Urbanisme et Reconstruction (éd.), « Sarrebruck ». La Sarre. Urbanisme. 1946. Sarrebruck : 1947, p. 55

Pingusson eu maintes fois l'occasion de présenter ses plans pour la reconstruction de Sarrebruck, non seulement sur place, mais aussi à Paris (par exemple au Salon d'automne qui se tint sous l'égide de René Herbst, président de l'Union des artistes modernes, en septembre $\left.1948^{24}\right)$.

Le 14 juillet 1947, ses plans furent traduits et publiés une première fois dans Saar Städtebau $1946^{25}$, une publication qui, au premier regard, semble identique à la version française (La Sarre. Urbanisme. 1946). À y regarder de plus près cependant, on peut constater qu'il y manque en réalité des parties essentielles comme le chapitre "Plan » contenant le plan d'aménagement régional, et que les erreurs de traduction abondent. Début décembre 1947, le premier numéro de BAU (Construction), le magazine sarrois « de l'habitat, du travail et du repos ${ }^{26}$ ", édité par l'architecte Otto Renner, en publiera une version remaniée ${ }^{27}$. L'architecture et l'urbanisme sont présentés ici comme les clés d'une vie meilleure et comme les marqueurs d'une société moderne et démocratique. Pour la première fois, on peut y lire des extraits de la Charte d'Athènes en allemand: «1. Die Stadt ist ein Teil eines ökonomischen, sozialen und politischen Ganzen: des Gebiets (der Region) » («1. La ville fait partie d'un ensemble économique, social et politique, du territoire, de la région.»). L'éditeur conclut sur ces mots: "Möge die Zeitschrift BAU nun ihre Aufgabe erfüllen und zum Aufbau und zu der Gestaltung eines neuen Europa beitragen!» ("Que le magazine $B A U$ remplisse désormais sa mission et contribue à la construction et au développement d'une nouvelle Europe ! $)^{28}$. 


\section{Capitale de la CECA}

La situation centrale de l'ambassade dans le plan de la ville, son histoire et les progrès de sa construction et de son aménagement intérieur sont étroitement liés aux débuts de la CECA et au projet «Sarrebruck Capitale de la CECA» (fig. 7). Les premiers plans de situation, plans de masse et esquisses de la décoration intérieure de l'ambassade ont été établis en juillet et août 1950, à peine deux mois après la présentation par Robert Schuman du projet d'une Communauté européenne du charbon et de l'acier, le 9 mai 1950 à Paris. Un premier plan d'exécution signé par les «architectes associés PingussonSchultheis-Baur » / " Architektengemeinschaft Pingusson-Schultheis-Baur » est daté de la fin de l'année 1950. Une deuxième mouture est élaborée en janvier 1951. Les architectes commentèrent leurs plans dans un texte rédigé en commun, un " projet descriptif de la construction ${ }^{29}$ » sur lequel allaient se fonder ensuite toutes les descriptions parues dans les journaux. La construction de l'ambassade et celle d'un nouveau centre-ville autour de l'édifice y apparaissaient comme les parties d'un seul et même programme de construction.

\section{Figure 7}

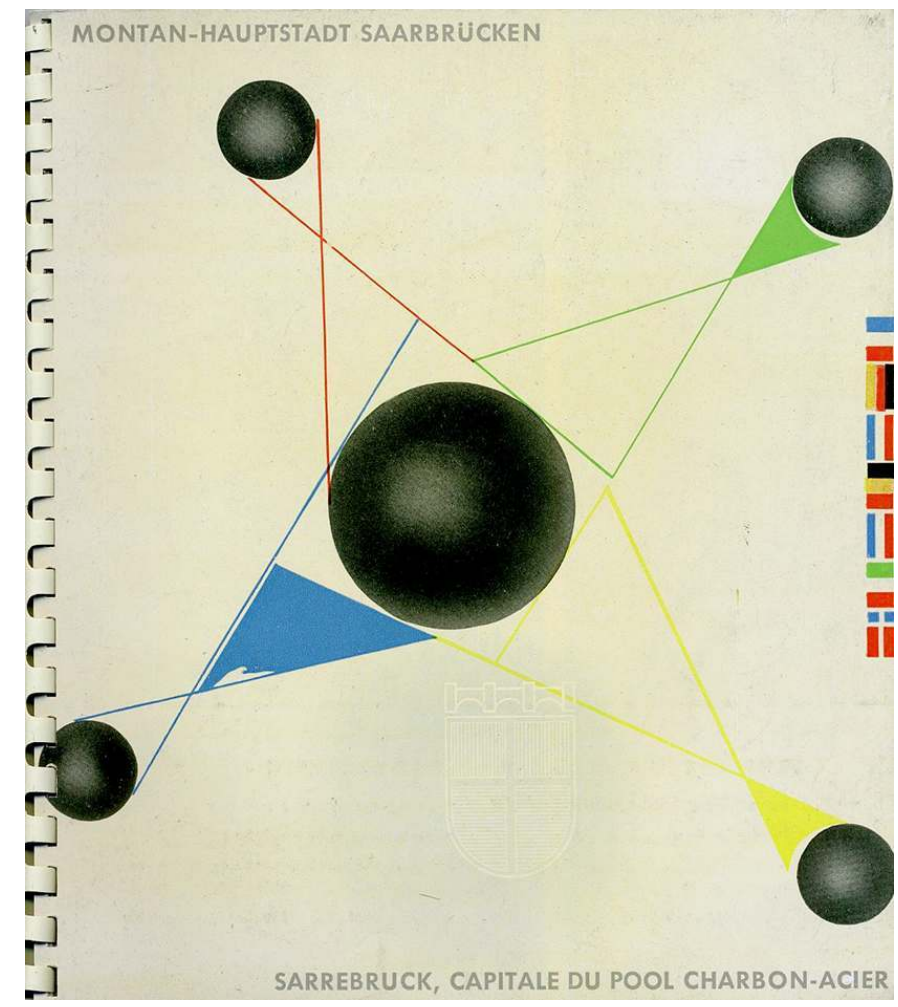

Couverture de la brochure «Sarrebruck, Capitale du Pool Charbon-Acier / Montan-Hauptstadt Saarbrücken », Ville de Sarrebruck (éd.). Sarrebruck : 1953.

En juin 1951, Sarrebruck se porta candidate pour devenir le «siège des institutions permanentes du Pool» auprès de la Haute Commission de la CECA, une instance supranationale. Le maire s'engagea à construire des bâtiments administratifs adaptés " sur des grands terrains propres à la construction en plein centre de la ville " ". Grandval développa dans ce contexte la vision d'un deuxième Luxembourg, un " "District of 
Columbia" de l'Europe » et il présenta "Sarrebrück [comme] le siège de toutes les Institutions européennes immédiates ou à venir ${ }^{31}$ ». Grandval fut nommé ambassadeur de France en Sarre le 25 janvier 1952. Peu de temps après, il demanda à Hoffmann de promouvoir le projet urbanistique autour de l'ambassade. La même année, Otto Renner, sur lequel on ne trouve aucune biographie ${ }^{32}$ alors qu'il joua pourtant un rôle éminent dans la candidature de Sarrebruck pour devenir capitale de la CECA dans les années 1950-1955, développa sa propre vision. Même si celle-ci ne s'imposa pas, elle reste intéressante : il s'agissait d'installer dans l'environnement immédiat de l'ambassade le cœur de la Haute Autorité de la CECA qui aurait abrité l'«assemblée générale», le «Comité spécial des ministres » et la «Cour de justice $»^{33}$.

\section{L'ambassade}

L'édifice était conçu, d'après la description des architectes, en deux «blocs » de hauteur différente, l'un dédié à l'administration (le consulat), l'autre à l'ambassade (fig. 8). Il consiste en une lame verticale de sept étages, de presque cent mètres de long et seulement huit mètres de large et de trois cubes horizontaux, regroupant les pièces de l'ambassade: réception, résidence et service. Pingusson avait déjà dessiné dans son « avant-projet » d'août 1950 de nombreuses esquisses pour l'aménagement de la partie dévolue à l'ambassade, pour l'entrée et les pièces d'habitation ou le bureau de l'ambassadeur qui relient les deux «blocs » du bâtiment. Finalement, ce sont Raphaël Raffel et Jacques Dumond, deux créateurs français bien établis, qui seront chargés de l'aménagement intérieur et qui feront du bureau de l'ambassadeur et des pièces de réception des vitrines de l'artisanat de luxe français.

Figure 8

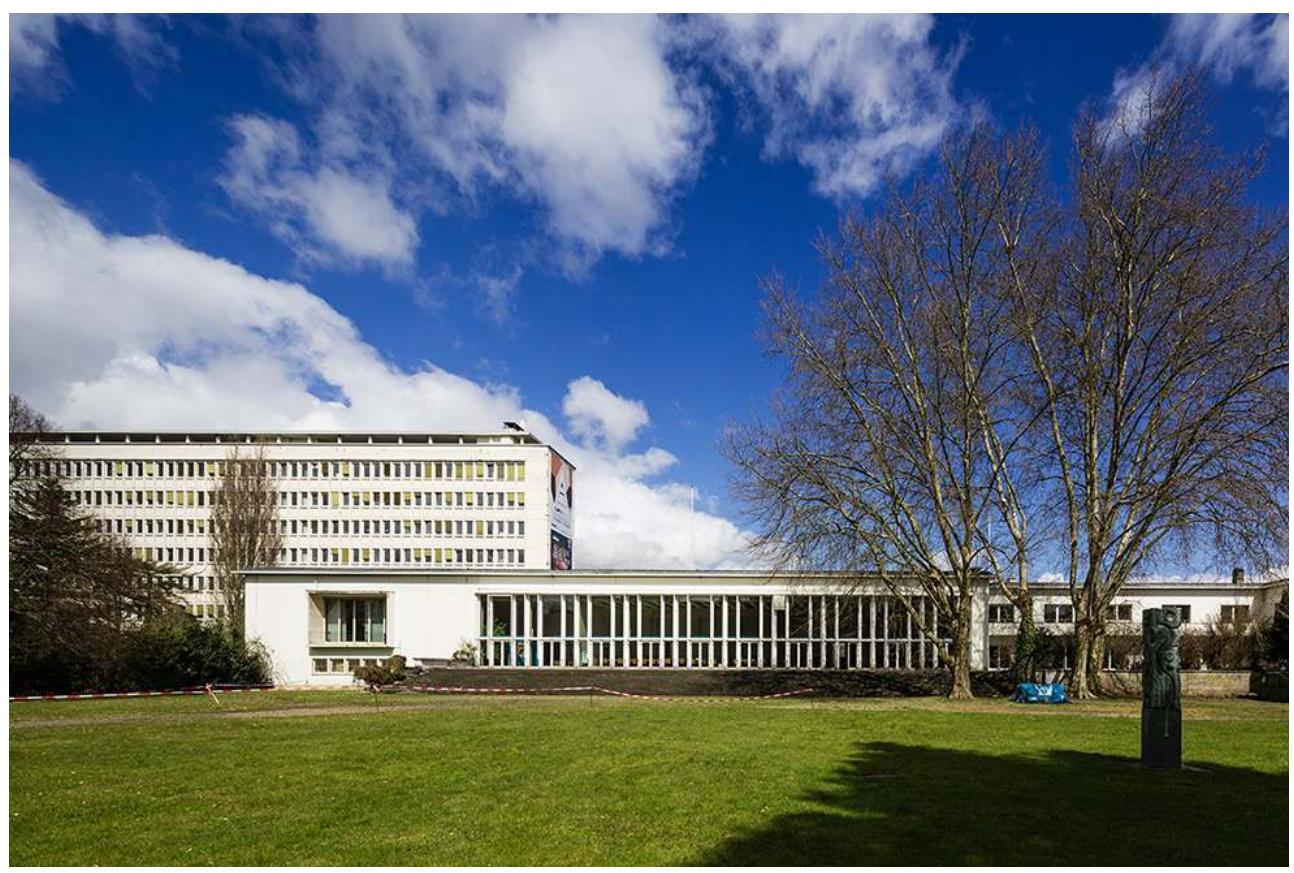

Ancienne ambassade de France, vue par le côté sud.

Phot. die arge lola, 2006. (c) die arge lola/regiofactum. 
bureaux et les pièces de réception de l'ambassadeur donnent sur le magnifique hall d'entrée et l'entrée principale est mise soigneusement en valeur par une «cour d'honneur » qui constitue le cœur de l'ensemble de la composition (fig. 9).

Figure 9

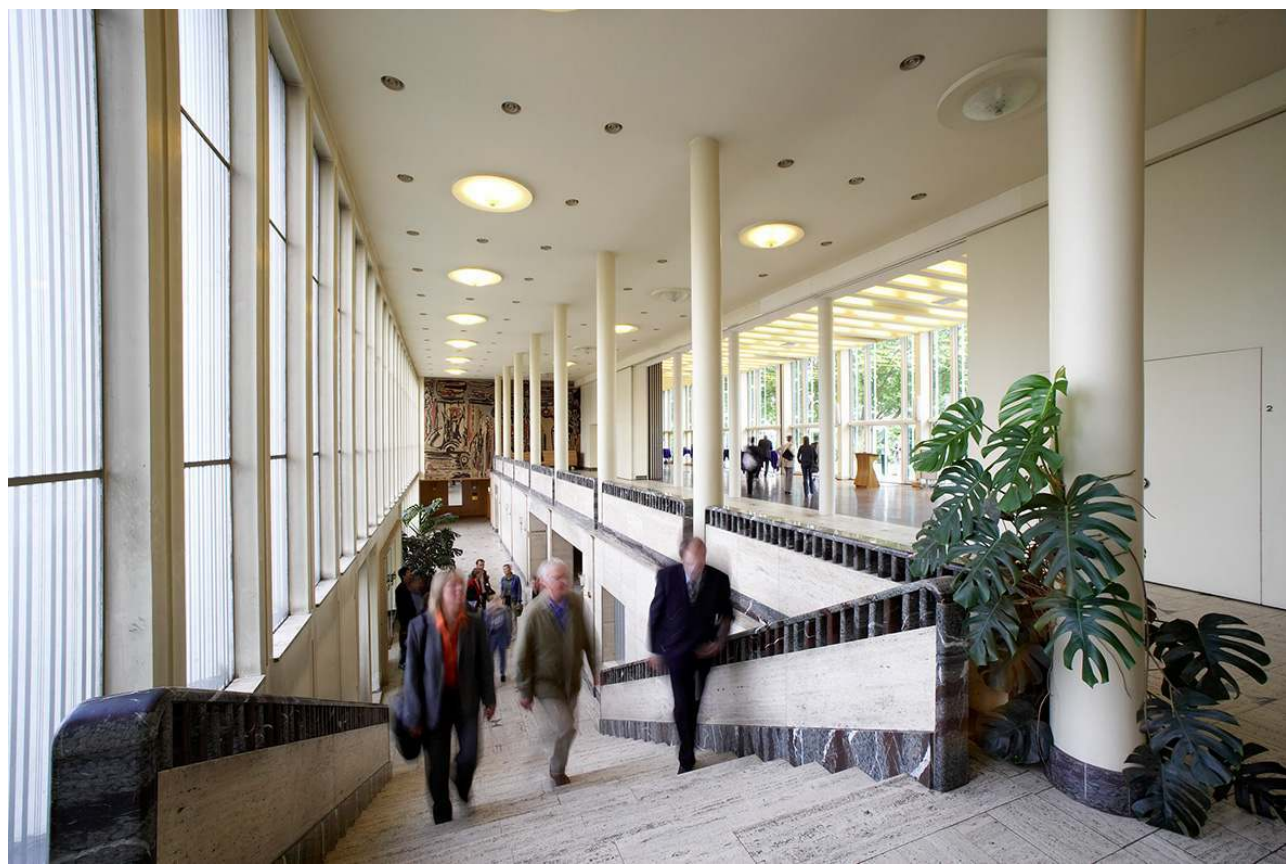

Ancienne ambassade de France, entrée par la cour d'honneur, vue dans la galerie.

Phot. die arge lola, 2006. (c) die arge lola/regiofactum.

Malgré la différenciation des volumes, la circulation entre les différents corps de bâtiment est fluide: les plans horizontaux et verticaux, les espaces intérieurs et extérieurs s'interpénètrent de manière organique. Certains espaces sont même modulables, tels les bureaux dans le bâtiment haut ou la salle de réception : ses élégantes portes articulées, d'une hauteur inhabituelle, peuvent, si besoin, ouvrir la pièce sur le hall d'entrée. Quelques-uns des meilleurs représentants de l'art moderne et du design ont travaillé à ce projet.

De nombreux éléments d'origine ont été conservés et témoignent du faste de l'ancienne ambassade, comme les tapisseries monumentales de 75 mètres carrés, conçues sur mesure en France par François Arnal, que l'on peut encore admirer dans le hall d'entrée, ou le dessin, lui aussi monumental, d'un paysage industriel de Claude Maurel, à la manière de Piranèse, ainsi qu'un relief (« sgrafitto ») de Otto Lackenmacher.

Un ensemble de détails, moins spectaculaires au premier regard et pourtant pensés tout aussi soigneusement, ne doivent pas être oubliés, comme les luminaires de Georges Lévy Mazaud et de Hans Bert Baur, les poignées de porte et autres éléments de quincaillerie (fig. 10) ou les peintures murales de Boris Kleint ${ }^{34}$ et une sculpture de l'artiste verrier Henri Navarre, qui malheureusement n'ont pas été conservées. 
Figure 10

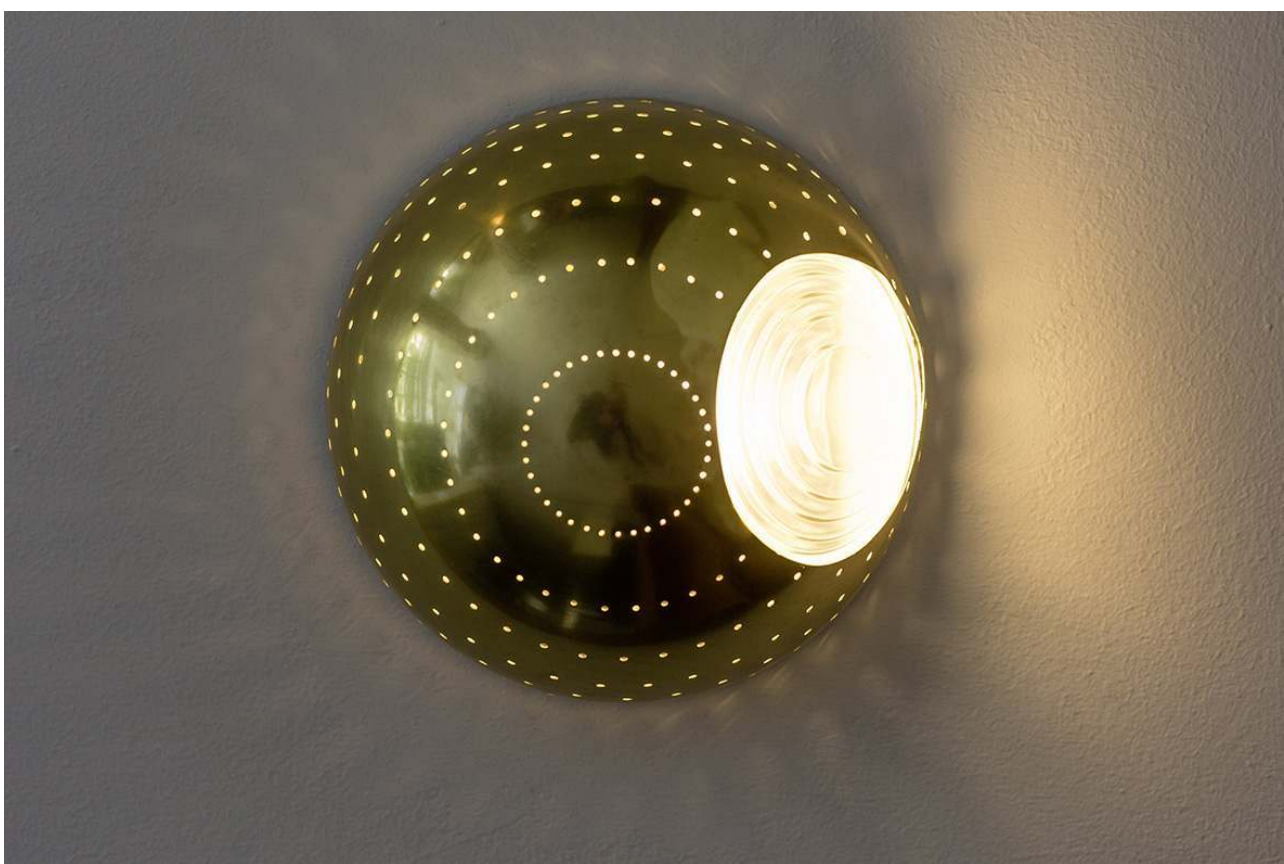

Ancienne ambassade de France, Spot.

Phot. die arge lola, 2013. (c) die arge lola/regiofactum.

Les architectes ont construit un symbole de "l'esprit contemporain" tel qu'ils l'entendaient (souci de transparence, rôle de l'artisanat et respect du matériau).

Si « la planification ouverte » de l'ambassade rappelle celle du bâtiment du Bauhaus de Walter Gropius à Weimar et son projet d'Académie philosophique internationale ${ }^{35}$, son ameublement, surtout le «bloc » ambassade, s'inscrit pleinement dans la tradition des arts décoratifs et de l'artisanat de luxe français ${ }^{36}$.

En 1954, le bloc de l'ambassade (le «bloc I ») est doté de son précieux ameublement sans que cela ne fasse l'objet de mention particulière dans la presse de l'époque. En 1955, en revanche, L'Architecture d'aujourd'hui et un numéro spécial bilingue de Nature et technique, édité par Otto Renner, sont consacrés à "la Sarre et à sa mission européenne » et à l'« Ambassade de France en Sarre à Sarrebruck », en l'occurrence au " nouveau bâtiment de l'Ambassade de France à Sarrebruck ». Tandis que dans la revue française, on peut lire que Pingusson est le maître d'œuvre du projet architectural, Nature et technique, en revanche, présente «les architectes Pingusson, Schultheis, Baur» comme les responsables de la « planification et la mise en œuvre ${ }^{37}$ ». Dans les deux cas toutefois, c'est le bâtiment haut, bien visible de la Sarre, qui retient l'attention (fig. 11). 
Figure 11

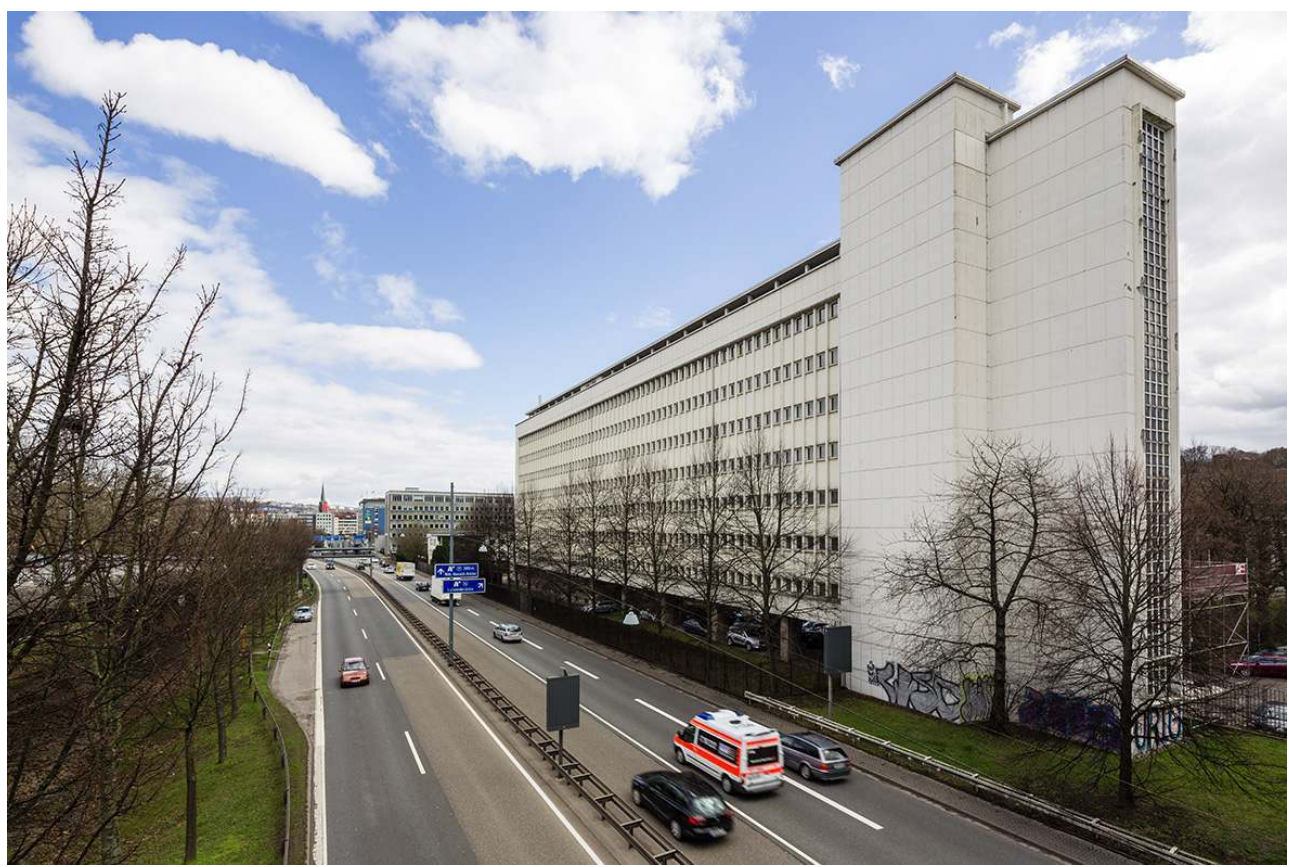

Ancienne ambassade de France, bâtiment haut (administration) avec la « Westspange », une bretelle d'accès à l'autoroute construite en 1986.

Phot. die arge lola, 2013. ( ) die arge lola/regiofactum.

Il n'est pas anodin, dans ces circonstances, que la partie du bâtiment dévolue à l'ambassade proprement dite soit justement celle qui fut la plus instrumentalisée par les partis nationalistes et soumise à une propagande virulente qui se manifesta avant le référendum. En août 1955, un article paru dans Deutsche Saar Zeitung intitulé « Grand luxe dans le palais de l'ambassadeur » dénonçait ainsi l'usage de "bois nobles et de marbre», la présence de «Gobelins et de tapis persans d'une valeur de plusieurs millions » et soulignait l'existence d'un étang à poissons, d'une serre, d'une piscine chauffée : « Le luxe mirifique du palais de l'ambassadeur a de quoi faire enrager le contribuable sarrois ${ }^{38}$." Quelques années plus tôt déjà, Grandval, «le gouverneur de la Sarre » et le " gouvernement d'exilés » dirigé par Hoffmann avaient été l'objet de violentes attaques dans la presse, en Allemagne comme en France, de la part de ceux qui refusaient l'autonomie de la Sarre.

Hans Bert Baur, Gilbert Grandval et Johannes Hoffmann achevèrent ensemble le projet de l'ambassade en janvier ou juin 1955. L'ensemble s'élevait à un montant total de « 880000000 francs français " ${ }^{39}$. Il n'est pas possible de déterminer ce qui devait être réellement pris en charge par le gouvernement de la Sarre et la France.

Tandis que l'étoile de Pingusson pâlissait très rapidement, la réputation de Grandval se dégrada au point de faire de lui un symbole de l'échec global du projet politique que Pingusson, Hoffmann, Grandval et Robert Schuman avaient voulu porter pour la Sarre. Les calomnies visaient à disqualifier l'ensemble d'une démarche dont la dimension culturelle et européenne contrevenait à l'évidence aux intérêts économiques des pays concernés. Le chancelier Konrad Adenauer ne déclara-t-il pas devant le Parlement allemand, en 1952, que l'Allemagne (la République fédérale) n'avait aucun intérêt à un État sarrois indépendant? 
35 Avec la fin de l'autonomie de la Sarre, l'ambassade de Sarrebruck perdit, au propre et au figuré, sa fonction politique. Le journaliste franco-allemand Peter Scholl Latour (qui fut porte-parole du gouvernement sarrois de 1954 à 1955) décrit l'atmosphère d'alors dans le Saarbrücker Zeitung du 6 juin 1959 :

La construction audacieuse et élancée qui domine le port de Sarrebruck et que l'on surnomme « le mouchoir de poche » dans le langage populaire, va changer de main dans un futur proche. En hiver, les rangées de fenêtres éclairées devant les eaux sombres de la Sarre évoquaient la silhouette d'un paquebot. Bientôt, l'ancien équipage des représentants de la France à Sarrebruck quittera le navire. Ce qu'il adviendra du bâtiment érigé sur les rives de la Sarre, qui s'affirme aujourd'hui comme l'une des créations architecturales les plus réussies du paysage urbain de Sarrebruck, personne ne le sait encore. $[. . .]^{40}$. Le 5 juillet 1959, l'union douanière et monétaire avec la France prit fin et la réunification avec la République fédérale d'Allemagne fut actée. En 1960, l'ancienne ambassade devint le ministère de la Culture du Land de la Sarre.

En 1985, le service régional de la conservation du patrimoine (Staatliches Konservatoramt des Saarlandes) classa le bâtiment comme monument historique eu égard à « son intérêt historique, artistique, économique et particulièrement urbanistique. Le classement concerne le site dans son ensemble, les murs, les équipements (mobilier d'époque), ainsi que leur environnement ${ }^{41} »$.

Depuis le milieu de l'année 2014, le bâtiment est vide. La constante exposition des côtés nord et est du bâtiment à l'utilisation intensive de l'autoroute, construite de 1956 à 1963, puis en 1986, a contribué à sa dégradation (fig. 12). Les responsables n'ont pas encore proposé de nouvelle affectation qui pourrait correspondre à son importance culturelle et politique. Aujourd'hui, l'ambassade ressemble plus que jamais à un paquebot échoué. 
Figure 12

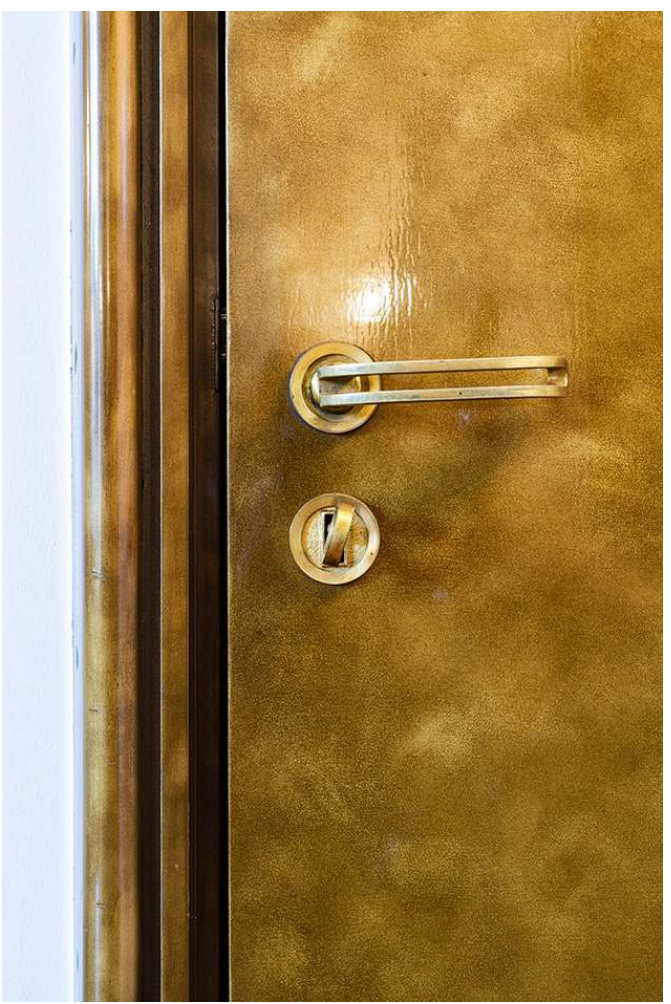

Porte du bureau de l'ambassadeur, ancienne ambassade de France en Sarre, Sarrebruck / Saarbrücken.

Phot. die arge lola, 2016. ( die arge lola/regiofactum.

\section{Pingusson « en Sarre »}

Les biographies de Baur, Schultheis ou Renner, nous l'évoquions plus haut, sont encore à écrire. Il est étonnant en outre qu'on ne trouve guère non plus d'étude sérieuse sur l'« œuvre sarrois » de Pingusson, qui fut pourtant l'un des architectes français les plus importants $\mathrm{du} \mathrm{xx}^{\mathrm{e}}$ siècle. Il fut membre de la rédaction de plusieurs revues spécialisées de renom, en particulier de L'Architecture d'aujourd'hui, et il collabora par ailleurs, avant et après la guerre, à divers mouvements culturels, tels le Congrès international d'architecture moderne (CIAM) ou l'Union des artistes modernes (UAM).

Parmi ses « travaux principaux », on compte " en 1945, urbanisme et reconstruction de la Sarre, plan de Sarrebrück et de sa région (en cours de réalisation), ambassade de France à Sarrebrück ${ }^{42}$ ». Dans le même temps Pingusson fut jusqu'en 1963 "chargé des plans d'aménagement du bassin houiller de Lorraine et de la région sidérurgique - cités nouvelles - architecte conseil du ministère de la construction depuis 1945 en Moselle ${ }^{43}$ ». En juillet 1949, au VII ${ }^{\mathrm{C} I A M}$ de Bergame, Pingusson obtint la médaille d'or pour son plan de reconstruction de Sarrebruck. En décembre 1951, il participa, en qualité de membre du jury, à la sélection des projets visant à faire de l'université un centre de formation européen. En 1955, il fut membre du jury du concours architectural international visant à faire de Sarrebruck la capitale de la CECA. Des années plus tard, alors qu'il avait déjà quitté la région, il rêvait encore d'une solidarité «sarro-lorraine » ${ }^{44}$, et continua 
d'entretenir des relations professionnelles avec le Luxembourg ou la Wallonie. Ce n'est pas un hasard s'il avait proposé dès 1947 dans La Sarre. Urbanisme. 1946 de compléter le plan régional pour la Sarre par des plans régionaux pour la Lorraine et le Luxembourg. La même année paraissait sa première biographie en langue allemande ${ }^{45}$. Pingusson travailla pour le gouvernement français, le gouvernement sarrois et la ville de Sarrebruck. Préservées sont ses correspondances en français comme en allemand. Comme nous l'avons vu, ses projets furent discutés au moins jusqu'en 1955. Sur ce point, les vues de Pingusson rejoignaient non seulement celles de Grandval, de Hoffmann ou de Peter Zimmer, également une figure importante de cette époque en sa qualité de maire de Sarrebruck et de président du Landtag (parlement) sarrois qui, tous, entretenaient des relations épistolaires avec Robert Schuman ${ }^{46}$. Elles s'accordaient aussi avec celles des habitants de la ville, comme le montre l'exemple de Dieter Heinz qui, en 1956, succéda à Pingusson dans l'ancien bureau que ce dernier occupait à l'hôtel de ville.

La Sarre fit de Pingusson, selon Dietmar Kolling, un européen de la première heure qui voulut contribuer à façonner le nouveau visage de la ville frontalière et industrielle de Sarrebruck et de sa région ${ }^{47}$.

La Sarre fait partie intégrante aujourd'hui de la Grande Région, qu'elle forme avec ses voisines, la Lorraine, le Luxembourg, la Rhénanie-Palatinat et la Wallonie (fig. 13). En 2007, cette région frontalière fut capitale européenne de la Culture. Cet événement plaça pour la première fois ce "paysage frontalier» sous le feu des projecteurs, en le présentant comme un espace transnational et mouvant où se rencontrent différentes aires linguistiques, dans un ensemble polycentrique, structuré autour de plusieurs petites "métropoles» et d'un patrimoine culturel et architectural qui porte la trace des échanges et des tensions politiques et culturels qui ont marqué cette région-frontière. $\mathrm{Si}$ l'on considère que $30 \%$ des Européens vivent dans des zones-frontière, on comprend mieux les enjeux d'une telle histoire, encore largement inexplorée, qui permet de regarder autrement notre passé. Pour saisir un lieu-frontière, par définition métis, il faut d'autres instruments conceptuels et langagiers que ceux dévolus à la description d'espaces nationaux ${ }^{48}$. 


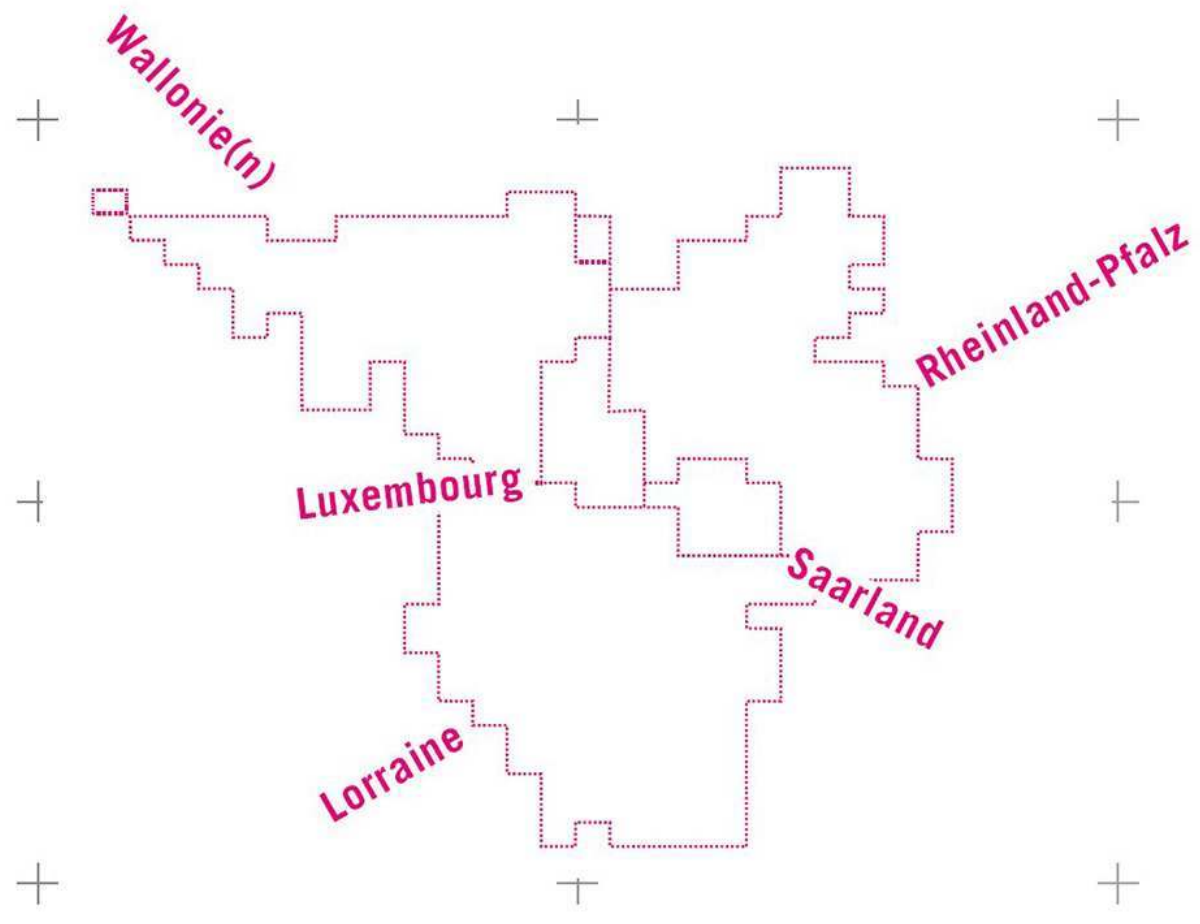

La Grande Région Sarre-Lorraine-Luxembourg-Rhénanie-Palatinat-Wallonie.

(c) regiofactum.

L'existence même de la Grande Région qu'est devenue cette zone frontalière permet, avec le recul, de porter un regard différent sur la période de 1945 à 1955. Elle offre une occasion inespérée de mettre en avant des points de vue qui ne se réduisent pas à des perspectives nationales et de re-découvrir des figures qui ont œuvré, à leur époque et à leur niveau local, à la construction de l'Europe.

Que les projets de Pingusson n'aient été que partiellement réalisés ou qu'ils soient tombés dans l'oubli, quand ils n'ont pas été carrément traités par le mépris, ne change rien au fait qu'il a porté la vision d'une communauté interculturelle et supranationale qui reste vivace aujourd'hui dans le modèle de la Grande Région et des relations que la Sarre entretient avec ses voisins.

Au-delà, son exemple a aussi une valeur paradigmatique pour la difficulté de mettre en œuvre des projets interculturels: plus que d'autres, ceux-ci doivent sans cesse être défendus, remis sur le métier car rien n'est jamais acquis. Il faut pour cela des citoyens engagés qui soient à la fois prêts à porter un regard différent sur l'architecture de l'aprèsguerre et désireux de partager l'héritage qu'ils ont en commun, comme nous y invite en 2018 l'European Cultural Heritage Year ${ }^{49}$ (fig. 14). 


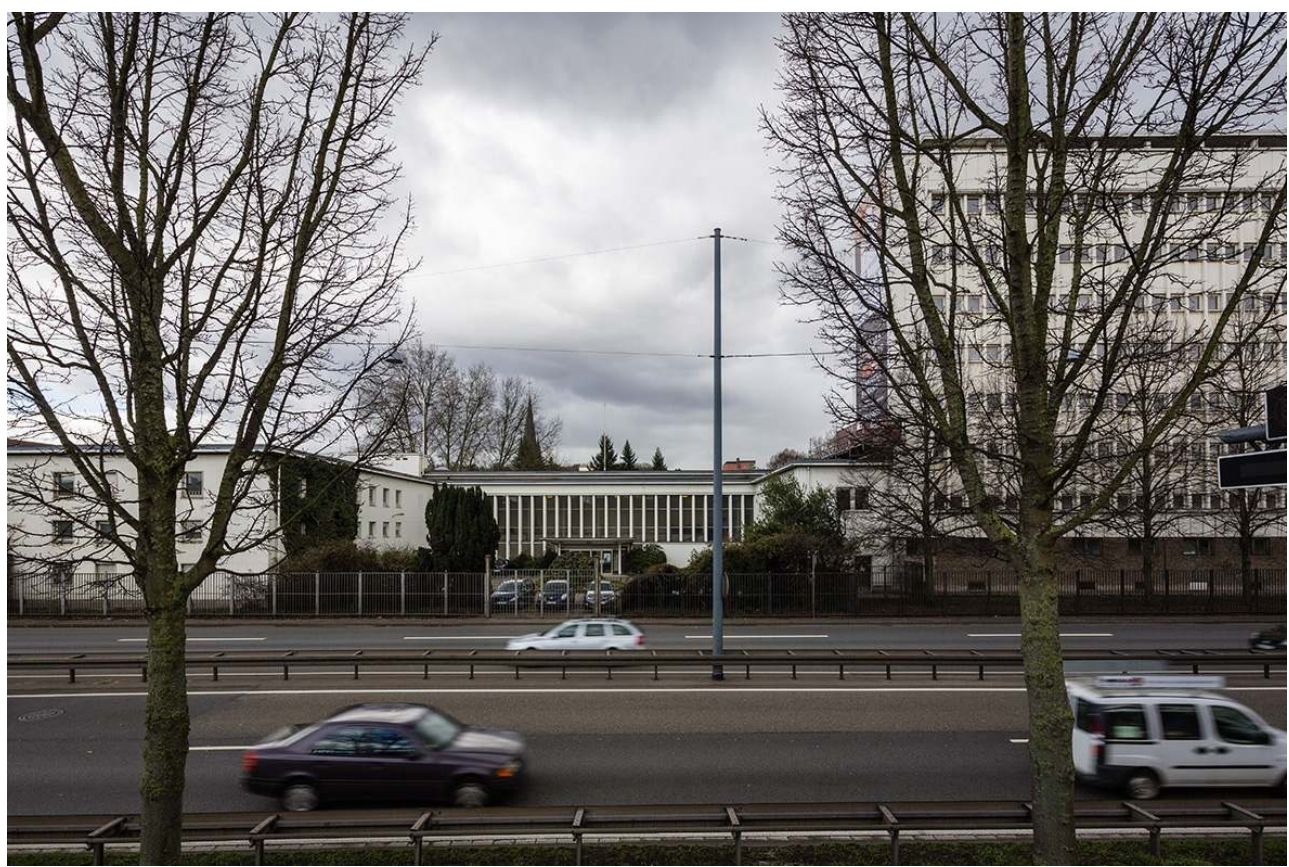

Ancienne ambassade de France, cour d'honneur coupée par l'autoroute, Haut-Bâtiment (Administration)

Phot. die arge lola, 2013. (c) die arge lola/regiofactum.

Bien qu'au cours des dernières années, quelques publications aient été consacrées à l'ambassade ${ }^{50}$, il manque encore une étude comparative de fond qui prenne en compte la littérature secondaire aussi bien en langue allemande qu'en langue française et que se fonde sur des recherches dans les archives en Sarre, en Allemagne et en France. Une analyse scientifique des bâtiments selon des critères artistiques et d'histoire de l'architecture, par exemple, fait toujours défaut. De même, manque la traduction des sources primaires dans les deux langues.

Les moyens alloués à des projets de recherche aussi complexes sont réduits, même lorsqu'ils ont une dimension européenne, et ils ne produisent pas forcément de résultats sur le court terme.

Faire de l'ambassade de France à Sarrebruck le symbole d'une "victoire de l'esprit français $»^{51}$ est une vision aussi réductrice que celle qui considère l'émancipation politique et culturelle initiée depuis 1945 comme un échec : le 23 octobre 1955 en effet, 67,7 \% des Sarrois votèrent, à la surprise générale, contre le statut européen de leur État et en faveur de la réunification avec l'Allemagne. Cette vision fataliste de l'histoire, empreinte de préjugés tenaces, s'oppose étrangement à la vision constructive de cette région défendue à l'époque par Robert Schuman et ses «alliés " - surtout Pingusson, Grandval, Hoffmann et Renner - dans la cause de l'Europe. C'est aussi l'un des freins idéologiques qui déforment la perception que l'on peut avoir de cette époque ${ }^{52}$. 


\section{NOTES}

1. - Gouvernement militaire de la Sarre, section Urbanisme et Reconstruction (éd.). La Sarre. Urbanisme. 1946. Sarrebruck : 1947, p. 97.

2. - Architecture d'aujourd'hui, 1945, $\mathrm{n}^{\circ} 1$, p. 10 : «Essai de Réorganisation Européenne avec le Planning moderne » : « Les pays modernes bilingues de la frontière franco-allemande s'inscrivent presque exactement dans les anciennes limites de la Lotharingie.»

3. - BRAUDEL, Fernand. L'Identité de la France. Paris : Flammarion, 1980-1986, t. 1, Espace et histoire, p. 17.

4. - GERGEN, Thomas. «Von der Saarprovinz zum Saarland. Die Vorgängerorganisationen des Saarlandes bis zu den Volksabstimmungen von 1935 und 1955 ». Saarländische Kommunalzeitschrift, $\mathrm{n}^{\circ}$ 9, 2005, p. 211-230.

5. - DAYDÉ, Cyril. Zone française d'occupation en Allemagne et en Autriche (ZFO). Archives des administrations françaises et des organismes tripartites, 1945-1955. Notice générale d'orientation et d'aide à la recherche. La Courneuve : ministère des Affaires étrangères, direction des Archives, Centre des Archives diplomatiques de LaCourneuve, mai 2013.Voir sur le site: https:// www.diplomatie.gouv.fr/IMG/pdf/zfo_fiche-de-recherche_v2_cleof2c21.pdf [consulté le 25/01/2019].

6. - Communauté européenne du charbon et de l'acier.

7. - Stadt Saarbrücken / Ville de Sarrebruck (éd.). Montan-Hauptstadt Saarbrücken/Sarrebruck, Capitale du Pool Charbon-Acier. Sarrebruck : 1953, non paginé.

8. - À ce propos, voir MENDGEN, Eva. «Über die Grenzen hinaus». Dans ZIMMERMANN, Olaf (dir.). Die dritte Säule: Beiträge zur Auswärtigen Kultur-und Bildungspolitik. Berlin: Deutschen Kulturrat, 2018, p. 369-370.

9. - ECKHARDT, Werner. Die Saar fließt nach Europa. Francfort-sur-le-Main : Verlag der Frankfurter Hefte, 1954, p. 178.

10. - MENDGEN, Eva. «Ex-Ambassade, die Botschaft Frankreichs an der Saar ». Saarbrücker Hefte, 2014, p. 90-96.

11. - Le Neues Bauen (dit aussi "Neue Bewegung", "Neue Sachlichkeit») est un courant architectural, version allemande du mouvement moderne, un réformisme architectural introduit par le Deutscher Werkbund et le Bauhaus. Ce mouvement est volontiers associé à Le Corbusier, Walter Gropius, Mies van der Rohe, Max Taut ou Pier Luigi Nervi. Voir par exemple WINKLER, Klaus-Jürgen. Bauhaus und Neues Bauen. Dans : Nationalatlas Bundesrepublik Deutschland. Leipzig/ Heidelberg : Institut für Känderkunde, Spektrum Akad. Verl., 2002, p. 154-155. Voir sur le site : http://archiv.nationalatlas.de/wp-content/art_pdf/Band6_154-155_archiv.pdf [consulté le 25/01/2019].

12. - Saarländischer Rundfunk, Interview Axel Buchholz-Eva Mendgen, $1^{\mathrm{er}}$ mai. 2016: Schloss Halberg: ein heraus-ragender europäischer Ort. Voir sur le site : https://www.sr.de/sr/home/der_sr/ wir_uber_uns/geschichte/fundstucke/20160501_fundstueck_mai2016_schloss_halberg100.html / ; BURGARD, Paul. «Die Schlösser des Monsieur Grandval. Teil 1: Die Metamorphosen des Halbergs ». SaarGeschichten, 2017, $n^{\circ} 1$, p. 20-35.

13. - Paris, Cité de l'architecture et du patrimoine (CAPA), Centre des archives d'architecture du $\mathrm{xx}^{\mathrm{e}}$ siècle, fonds Pingusson, dossier Ambassade, PINGU-H-50-2, 117.

14. - Voir sur le site: http://www.dircost.unito.it/cs/pdf/19471215_germaniaSaarland_ted.pdf [consulté le 13/02/2019].

15. - Voir le site : www.saar-nostalgie.de [consulté le 25/01/2019]. 
16. - Gouvernement militaire de la Sarre, section Urbanisme et Reconstruction (éd.). La Sarre. Urbanisme. 1946, achevé d'imprimer en mai 1947 à Sarrebruck sur les presses de la MalstattBurbacher Handelsdruckerei sous le contrôle technique de l'Imprimerie nationale, 100 pages. L'étude a été réalisée par l'équipe des urbanistes de la Sarre. Elle a été tirée à 1200 exemplaires. Voir notamment les p. 96-99.

17. - D'après HEINZ, Dieter. Zum Wiederaufbau in Saarbrücken. Manuscrit inédit, archives privées.

18. - PINGUSSON, Georges-Henri. L'Urbanisme de notre temps. Manuscrit inédit, 1952 (Centre des archives d'architecture $\mathrm{du} \mathrm{xx}^{\mathrm{e}}$ siècle, fonds Pingusson, Pièces personnelles et professionnelles).

19. - PINGUSSON, G.-H. «Urbanisme en Sarre ». Architecture d'aujourd'hui, nov.-déc. 1948, n² 21, p. 3.

20. - MENDGEN, Norbert. "L'Urbanisme : utopie et réalité à l'exemple de Sarrebruck ». Dans LIECHTENHAN, Francine-Dominique (dir.). Europe 1946. Entre le deuil et l'espoir. Bruxelles/Paris : Éd. Complexe, 1996, p. 199-212.

21. - FEIEN, Willi. «Der Wiederaufbau der Stadt Saarbrücken. Eine städtebauliche Plauderei ». Saarbrücker Hefte, 1955, 1-2, p. 9.

22. - PINGUSSON, Georges-Henri. Manuscrit de l'article "Ambassade française à Sarrebruck » paru dans Architecture d'aujourd'hui, nº 4, 1953 (Centre des archives d'architecture du xx siècle, fonds Pingusson, Pièces personnelles et professionnelles).

23. - « Le terrain se trouve placé le long de la Sarre et de ses berges aménagées en jardins publics à l'angle de l'axe Nord-Sud de la ville et de l'allée qui borde la Sarre (Saarufer)». "Celle-ci prolonge l'allée centrale du parc du château où sont situés les bâtiments administratifs de la ville, notamment le Landtag. L'Ambassade formera ainsi le fond de la perspective ouverte depuis la Place Centrale de Sarrebruck. " (PINGUSSON, G.H. "Ambassade française à Sarrebruck». Architecture d'aujourd'hui, $\mathrm{n}^{\circ} 4,1953$, p. 82).

24. - «Dépenses effectuées par M. Pingusson pour les travaux de la maquette de la ville de Sarrebruck pour l'exposition au Salon d'Automne à Paris, septembre 1948 » (Bilan) (Centre des archives d'architecture du $\mathrm{xx}^{\mathrm{e}}$ siècle, fonds Pingusson, Pièces personnelles et professionnelles).

25. - Gouvernement militaire de la Sarre, section Urbanisme et Reconstruction (éd.). Die Saar. Städtebau. 1946. Sarrebruck: Malstatt Burbacher Handelsdruckerei/Heiner Hantschke unter technischer Kontrolle der Imprimerie Nationale, juillet 1947.

26. - BAU Zeitschrift. Wohnen-arbeiten-sich erholen.

27. - PINGUSSON, Georges-Henri. «Projekt für Saarbrücken, Nachdruck aus Die Saar Städtebau ». $B A U$, décembre 1947, $\mathrm{n}^{\circ} 1$, p. 37-51.

28. - BAU-Zeitschrift. Wohnen, arbeiten, sich erholen, $1^{\text {er }}$ déc. $1947, \mathrm{n}^{\circ} 1$, S. 4 . Autorisée par le Gouvernement militaire de la Sarre - Direction de l'information, Nr. 3174, Inf. 5.3.1947. La revue sarroise a été soutenue par les revues françaises suivantes: L'Architecture d'aujourd'hui, L'Homme et l'architecture, Techniques et architecture.

29. - Tapuscrit, "AMBASSADE DE FRANCE EN SARRE - "Projet descriptif de la construction”, “ Sarrebruck, le 20 janvier 1951: PINGUSSON”, SCHULTHEIS, BAUR Architectes associés. " (Centre des archives d'architecture du $\mathrm{xx}^{\mathrm{e}}$ siècle, fonds Pingusson, dossier Ambassade).

30. - Lettre de Peter Zimmer, maire de Sarrebruck, à Robert Schuman, président de la conférence de la Communauté européenne du charbon et de l'acier, ministre des Affaires étrangères de la République française, Quai d'Orsay, Paris, daté de Sarrebruck, le 9 juin 1951 (Landesarchiv des Saarlandes, Sarrebruck).

31. - SCHNEIDER, Dieter Marc. "Gilbert Grandval Frankreichs Prokonsul an der Saar 1945 1955 ». Dans MARTENS, Stefan (dir.). Vom "Erbfeind" zum "Erneuerer". Aspekte und Motive der französischen Deutschlandpolitik nach dem Zweiten Weltkrieg. Sigmaringen: Thorbecke, 1993, p. 201-243, note 134 .

32. - Voir HEIN, Carola. The Capital of Europe. Architecture and Urban Planning for the European Union. Westport : Praegerd, 2004, p. 43-53. 
33. - Édition spéciale de Bau-Anzeiger für das Saarland comprenant un avant-propos du maire de Sarrebruck, $5^{\mathrm{e}}$ année, Sarrebruck : 1952.

34. - Foyer de la cantine et bar (dit aussi salle de la bibliothèque, détruit). Conception artistique. Dans : MENDGEN, Norbert. «L'Urbanisme : Utopie et Réalité à l'Exemple de Sarrebruck ». Dans LIECHTENHAN, Francine-Dominique (dir.). Europe 1946. Op. cit., p. 199-212.

35. - GIEDION, Siegfried. Raum, Zeit, Architektur [1941]. Ravensbourg : Otto Maier, 1965, p. 311-312. 36. - Il faut noter qu'il subsiste un grand nombre d'éléments utilisés dans l'aménagement intérieur. Mais en l'absence d'un « Raumbuch » et d'inventaire, il est aujourd'hui très difficile de reconstituer leur histoire et d'identifier leurs créateurs.

37. - RENNER, Otto. «Der Neubau der französischen Botschaft in Saarbrücken / Le nouveau bâtiment de l'Ambassade de France à Sarrebruck ». Natur und Technik, 1955, nº 4, p. 56-57.

38. - Anonyme. Deutsche Saar Zeitung, août 1955 (Stadtarchiv Saarbrücken, 79995 55-64/7911).

39. - Cela correspond au devis estimatif des architectes du 27 juin 1952, accepté par le gouvernement de la Sarre. Les deux lettres sont conservées au Landesarchiv des Saarlandes, Sarrebruck.

40. - SCHOLL-LATOUR, Peter. "Das, schmale Handtuch" wechselt seine Bewohner - Aus der französischen Delegation wird das wichtigste Generalkonsulat auf deutschem Boden». Saarbrücker Zeitung, 6 juin 1959.

41. - Lettre de Norbert Mendgen, conservateur régional, au ministre de la Culture, de l'Éducation et de la Science du Land de la Sarre du 23 juillet 1985, coll. privée. En dépit de la protection dont il fait l'objet, le bâtiment a souffert, à tel point que le ministère de la Culture a dû déménager en 2014.

42. - PINGUSSON, Georges-Henri. Biographies, manuscrit (Centre des archives d'architecture du $\mathrm{xx}^{\mathrm{e}}$ siècle, fonds Pingusson, Pièces personnelles et professionnelles).

43. - Ibidem.

44. - Voir Société d'histoire et d'archéologie de Lorraine (éd.). Urbanisme et architecture en Lorraine. 1830-1930. Metz: Serpenoise, 1982, p. 36 : « La France considéra, en 1918 ainsi qu'en 1945, qu'elle n'avait qu'une fenêtre sur l'Allemagne : l'Alsace. Or il y avait une deuxième, la Moselle. Mais cela on l'ignore à Paris. La Lorraine fut toujours considérée comme une région marginale de l'espace français, ce qui géographiquement est exact. Mais nos politiciens, à de rares exceptions près, et nos aménageurs, n'ont jamais osé constater la réalité, à savoir que la Lorraine a une position centrale par rapport à l'Europe de l'Ouest. "

45. - BAU, décembre 1947, $\mathrm{n}^{\circ}$ 1, p. 79 : „Biographische Daten der Autoren“: „Georges-Henri Pingusson, geb. 1894, studierte, nach Teilnahme am ersten Weltkrieg, an der École Nationale des Beaux-Arts in Paris (Diplom im Jahre 1925). Studienreisen in Italien, Spanien und USSR. Viele Bauten, darunter Gebäude in Paris und Vichy, Lichtspielhäuser, Villen in Cannes, St.Jean de Luz, Elektrizitätsanlage in Vitry s. Seine, das

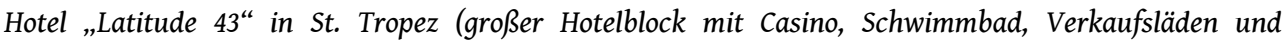
Garagen). Projekte zur Neubebauung von Paris, St. Girons, des Flughafens von Le Bourget u.a.m. - Mitglied der UAM (Union des Artistes Modernes), der SAM (Société des Architectes Modernes) und der SAP (Société des Architectes Préfabricateurs)." (Georges-Henri Pingusson, né en 1894, après avoir participé à la Première Guerre mondiale, étudia à l'École nationale des beaux-arts à Paris (diplômé en 1925). Effectua des voyages d'études en Italie, Espagne, et URSS. On lui doit de nombreuses réalisations à Cannes et Vichy, des cinémas et des villas à Cannes et Saint-Jean-de-Luz, l'usine électrique de Vitry-sur-Seine, l'hôtel Latitude 43 à Saint-Tropez (grand hôtel avec casino, piscine, boutiques et garages). Projets de nouvelles constructions à Paris, Saint-Girons, l'aéroport du Bourget Membre de l'UAM (Union des artistes modernes), de la SAM (Société des architectes modernes) et de la SAP (Société des architectes préfabricateurs).

46. - Correspondances inédites conservées aux Archives de la Sarre, Sarrebruck. 
47. - KOLLING, Dietmar. «Die Französische Botschaft und der Aufbauplan von Georges-Henri Pingusson für Saarbrücken » dans DEUTSCHER WERKBUND und INSTITUT FÜR AKTUELLE KUNST IM SAARLAND (éd.), Op. cit., p. 58-79.

48. - À ce sujet voir : MENDGEN, Eva, DOUCET, Hervé, HILDISCH, Volker (éd.). Im Reich der Mitte/ Le berceau de la civilisation européenne. Sarrebruck/Constance : Regiofactum/Hartung-Gorre, 2007 et MENDGEN, Eva (éd.). Au centre de l'Europe. Des liens et des lieux/Im Reich der Mitte 2. Kulturgemeinschaft Großregion. Constance : Hartung-Gorre/regiofactum 2013.

49. - «Sharing Heritage - Europäisches Kulturerbejahr». Deutsches Nationalkomitee für Denkmalschutz bei der Beauftragten der Bundesregierung für Kultur und Medien. Voir le site: https://sharingheritage.de/en/ [consulté le 25/01/2019].

50. - DEUTSCHER WERKBUND und INSTITUT FÜR AKTUELLE KUNST IM SAARLAND (éd.). Die ehemalige Französische Botschaft in Saarbrücken von Georges-Henri Pingusson. Ein Monument deutschfranzösischer Baukultur im Saarland / L'ancienne Ambassade de France à Sarrebruck de Georges-Henri Pingusson. Un monument de la culture architecturale franco-allemande en Sarre, Saarbrücken 2014. Voir: CARRIÉ, Benoît. «La construction de l'ancienne ambassade de France à Sarrebruck: le destin tumultueux d'un prototype devenu unicum », In Situ [En ligne], $34 \mid 2018$, mis en ligne le 23 mai 2018, consulté le 25 janvier 2019. URL : http://journals.openedition.org/insitu/16039 ; DOI : 10.4000/insitu.16039.

51. - Benoît Carrié, par exemple, interprète l'ambassade comme «un remarquable dispositif scénographique propice aux multiples mises en scène du pouvoir ", mais il n'entend par là que le pouvoir français. Il donne par ailleurs de la France une image selon nous réductrice pour ne pas dire convenue. La France devenant, dans son analyse, l'incarnation d'une « vision humaniste et cartésienne. »

52. - SCHUMAN, Robert. Pour l'Europe. Paris : 1963.

\section{RÉSUMÉS}

Cet article est consacré à un bâtiment qui fait partie du patrimoine non seulement architectural mais aussi politique et culturel de la Sarre, un héritage dont la portée reste largement méconnue. Vue à travers le prisme européen, l'ancienne ambassade de France en Sarre prend une dimension nouvelle comme témoin singulier d'une expérimentation à grande échelle sur la vocation européenne de cette région et terrain privilégié d'un nouveau départ qui devait permettre de surmonter les anciens combats idéologiques dans le cadre d'un "planning moderne » encore à définir. L'ancienne ambassade de France à Sarrebruck est le dernier vestige emblématique de la vocation européenne de ce petit État indépendant que fut la Sarre entre 1947 et 1955. L'édifice est le résultat d'une collaboration (dont l'histoire reste à faire) des "architectes associés » francosarrois Georges-Henri Pingusson, Bernhard Schultheis et Hans Bert Baur. Il a été conçu et érigé entre juillet 1950 et janvier 1955. Il occupe une position centrale dans le plan de la ville présenté par Pingusson, un des architectes en chef venu en Sarre avec le gouvernement militaire français en Sarre en octobre 1945. Son histoire et les progrès de sa construction et de son aménagement intérieur sont étroitement liés aux débuts de la CECA et au projet «Sarrebruck capitale de la CECA ». Cet article est la synthèse d'une recherche qui porte le même titre menée par l'auteure dans le cadre de l'École nationale supérieure d'architecture de Nancy et de l'université de Lorraine. Elle souhaite développer les réflexions liées à la définition d'un espace culturel, notamment la question de l'architecture et de l'urbanisme contemporains transfrontaliers, en 
prenant comme cas d'étude concret l'ancienne ambassade de France en Sarre en interrogeant les enjeux de mémoire qui se nouent autour du devenir actuel de l'édifice comme patrimoine partagé à l'échelle de la ville de Sarrebruck et de la Grande Région Sarre-Lorraine-LuxembourgRhénanie-Palatinat-Wallonie et la région Grand Est. Ce projet entend poursuivre une démarche commune rassemblant des chercheurs et des professionnels, des étudiants et leurs professeurs, de langues allemande et française, tout en montrant la difficulté d'établir un espace frontalier comme territoire culturel cohérent avec ses propres lois.

The subject of this essay is the former French Embassy in Saarbrücken, perhaps the most iconic building to represent the idealistic origins and the cultural components of the European Coal and Steel Community. It is situated on the left bank of the Saar River in the city centre. A bi-national team of three architects signed the building plans: the edifice was conceived and erected between 1950 and 1955 by the well-known French architect and urbanist Georges-Henri Pingusson and his two local associates, Bernhard Schultheis and Hans Bert Baur. At this time the Saarland was an independent state with a vocation to unify the states of the Coal and Steel Community, founded in 1950. Pingusson had already come to Saarbrücken in October 1945. He was in charge of the regional development plan for the Saar and the reconstruction and modernisation of Saarbrücken. He arrived with the French military government under Gilbert Grandval, who would later become French ambassador. In Pingusson's vision, the former bastion would be transformed into a link between countries. He even recommended corresponding development plans for neighbouring Lorraine and Luxemburg. When the ground was prepared for the candidature of Saarbrücken as a capital of the Coal and Steel Community, the building of the new French embassy set the aesthetic and cultural standards at this crossroads of Europe. Today the Saarland belongs to the Federal Republic of Germany, but it is also part of the 'Greater Region Saarland-Lorraine-Luxemb(o)urg-Rheinland-Pfalz-Wallonie(n)'. Under the auspices of this multinational region, the question of 'shared' or 'sharing' heritage is highly important. The Saar is no longer a steel and coal producing district, nor are her neighbours, and the French Embassy has lost its original function. Although it is a protected heritage site since 1985, parts of the building are threatened by decay and potential demolition. Valid arguments for its preservation will require fundamental cross-border research and cooperation, ideally in line with the comparative approach promoted long ago at the European University of the Saarland. It is time to practice a pluralistic perspective free from propaganda considerations to enable the rehabilitation not only of this particular building but also the cultural heritage of the entire Greater Region.

\section{INDEX}

Mots-clés : Allemagne, ambassade de France, André Remondet, après-guerre, architecte, archives, artiste, centre d'archives d'architecture du XXe siècle, centre des arts et métiers, Communauté européenne du charbon et de l'acier, identité, image, indépendant, inventaire, Georges-Henri Pingusson, maquette, matérialité, modèle 3 D, monument historique, plan, Sarrebruck, tapisserie, territoire français

Keywords : Germany, French embassy, André Remondet, post-war, architect, archives, artist, centre for the architectural archives of the twentieth century, 'centre des arts et métiers', European Coal and Steel community, identity, image, independent, inventory, Georges-Henri Pingusson, Hans Bert Baur, model, materiality, 3-D model, historic monument, plan, Sarrebruck, tapestry, French territory, UAM 


\section{AUTEUR}

\section{EVA MENDGEN}

Historienne de l'art et publiciste indépendante, fondatrice du réseau culturel de la Grande Région / Großregion Saarland-Lorraine-Luxemb(o)urg-Rheinland-Pfalz-Wallonie(n), docteur en histoire de l'art Rheinische-Friedrich-Wilhelms-Universität Bonn info@mendgen.com 